\title{
Local dimension is unbounded for planar posets
}

\author{
Bartłomiej Bosek* \\ Theoretical Computer Science Department \\ Faculty of Mathematics and Computer Science \\ Jagiellonian University in Kraków \\ Kraków, Poland \\ bosek@tcs.uj.edu.pl \\ Jarosław Grytczuk ${ }^{\dagger}$ \\ Faculty of Mathematics and Information Science \\ Warsaw University of Technology \\ Warszawa, Poland \\ j.grytczuk@mini.pw.edu.pl \\ William T. Trotter \\ Georgia Institute of Technology \\ Atlanta, Georgia, U.S.A. \\ trotter@math.gatech.edu
}

Submitted: Jan 2, 2020; Accepted: Oct 18, 2020; Published: Nov 13, 2020

(C) The authors. Released under the CC BY-ND license (International 4.0).

\begin{abstract}
In 1981, Kelly showed that planar posets can have arbitrarily large dimension. However, the posets in Kelly's example have bounded Boolean dimension and bounded local dimension, leading naturally to the questions as to whether either Boolean dimension or local dimension is bounded for the class of planar posets. The question for Boolean dimension was first posed by Nešetřil and Pudlák in 1989 and remains unanswered today. The concept of local dimension is quite new, introduced in 2016 by Ueckerdt. Since that time, researchers have obtained many interesting results concerning Boolean dimension and local dimension, contrasting these parameters with the classic Dushnik-Miller concept of dimension, and establishing links between both parameters and structural graph theory, path-width and tree-width in particular.

Here we show that the local dimension is not bounded in the class of planar posets. Our proof also shows that the local dimension of a poset is not bounded in terms of the maximum local dimension of its blocks, and it provides an alternative proof of the fact that the local dimension of a poset cannot be bounded in terms of the tree-width of its cover graph, independent of its height.
\end{abstract}

Mathematics Subject Classifications: 06A07, 05C35

* Supported by Polish National Science Center (NCN) grant 2013/11/D/ST6/03100.

${ }^{\dagger}$ Supported by Polish National Science Center (NCN) grant 2015/17/B/ST1/02660.

${ }_{\ddagger}^{\ddagger}$ Supported by a Simons Foundation Collaborative Research Grant. 


\section{Notation, Terminology and Background Discussion}

In this paper, we investigate combinatorial problems for finite posets. As has become standard in the literature, we use the terms elements and points interchangeably in referring to the members of the ground set of a poset. We will write $x \| y$ in $P$ when $x$ and $y$ are incomparable in a poset $P$, and we let $\operatorname{Inc}(P)$ denote the set of all ordered pairs $(x, y)$ with $x \| y$ in $P$. As a binary relation, $\operatorname{Inc}(P)$ is symmetric. Recall that a non-empty family $\mathcal{R}$ of linear extensions of $P$ is called a realizer of $P$ when $x<y$ in $P$ if and only if $x<y$ in $L$ for each $L \in \mathcal{R}$. Clearly, a non-empty family $\mathcal{R}$ of linear extensions of $P$ is a realizer of $P$ if and only if for each $(x, y) \in \operatorname{Inc}(P)$, there is some $L \in \mathcal{R}$ for which $x>y$ in $L$. The dimension of a poset $P$, as defined by Dushnik and Miller in their seminal paper [3], is the least positive integer $d$ for which $P$ has a realizer $\mathcal{R}$ with $|\mathcal{R}|=d$.

In recent years, researchers have been investigating combinatorial problems for two variations of the Dushnik-Miller concept for dimension, known as Boolean dimension and local dimension, respectively.

Here is the setting for Boolean dimension. For a positive integer $d$, we let $\mathbf{2}^{d}$ denote the set of all $0-1$ strings of length $d$. Such strings are also called bit strings. Let $P$ be a poset and let $\mathcal{B}=\left\{L_{1}, L_{2}, \ldots, L_{d}\right\}$ be a family of linear orders on the ground set of $P$ (these linear orders need not be linear extensions of $P$ ). Also, let $\tau$ be a function which maps all $0-1$ strings of length $d$ to $\{0,1\}$. For each pair $(x, y)$ of distinct elements of $P$, we form the bit string $q(x, y, \mathcal{B})$ which has value 1 in coordinate $i$ if and only if $x<y$ in $L_{i}$. We call the pair $(\mathcal{B}, \tau)$ a Boolean realizer of $P$ if for every pair $(x, y)$ of distinct elements of $P, x<y$ in $P$ if and only if $\tau(q(x, y, \mathcal{B}))=1$. Nešetřil and Pudlák [12] defined the Boolean dimension of $P$, denoted $\operatorname{bdim}(P)$, as the least positive integer $d$ for which $P$ has a Boolean realizer $(\mathcal{B}, \tau)$ with $|\mathcal{B}|=d$. Clearly, $\operatorname{bdim}(P) \leqslant \operatorname{dim}(P)$, since if $\mathcal{R}=\left\{L_{1}, L_{2}, \ldots, L_{d}\right\}$ is a realizer of $P$, we simply take $\mathcal{B}=\mathcal{R}$ and $\tau$ as the function which maps $(1,1, \ldots, 1)$ to 1 while all other bit strings of length $d$ are mapped to 0 .

Trivially, $\operatorname{bdim}(P)=1$ if and only if $P$ is either a chain or an antichain. We say that a poset $Q$ is a subposet of $P$ if for any two elements $x, y$ in $Q$ we have $x \leqslant y$ in $Q$ if and only if $x \leqslant y$ in $P$. So, if $Q$ is a subposet of $P$, then $\operatorname{bdim}(Q) \leqslant \operatorname{bdim}(P)$. In this paper, we will denote the dual of poset $P$ as $P^{*}$, and we define it as follows: the set of vertices of $P$ and $P^{*}$ are the same and $x \leqslant y$ in $P^{*}$ if and only if $y \leqslant x$ in $P^{*}$. Clearly, $\operatorname{bdim}(P)=\operatorname{bdim}\left(P^{*}\right)$. It is an easy exercise to show that if $\operatorname{bdim}(P)=2$, then $\operatorname{dim}(P)=2$. In [16], Trotter and Walczak prove the modestly more challenging fact that if $\operatorname{bdim}(P)=3$, then $\operatorname{dim}(P)=3$. As we will see shortly, for every $d \geqslant 4$, there is a poset $P$ with $\operatorname{bdim}(P)=4$ and $\operatorname{dim}(P)=d$.

Here is the setting for local dimension. Let $P$ be a poset. A partial linear extension, abbreviated ple, of $P$ is a linear extension of a subposet of $P$. Whenever $\mathcal{L}$ is a family of ple's of $P$ and $u \in P$, we set $\mu(u, \mathcal{L})=|\{L \in \mathcal{L}: u \in L\}|$. In turn, we set $\mu(P, \mathcal{L})=$ $\max \{\mu(u, \mathcal{L}): u \in P\}$. A non-empty family $\mathcal{L}$ of ple's of a poset $P$ is called a local realizer of $P$ if the following two conditions are satisfied: (1) If $x<y$ in $P$, there is some $L \in \mathcal{L}$ for which $x<y$ in $L$; (2) if $(x, y) \in \operatorname{Inc}(P)$, there is some $L \in \mathcal{L}$ for which $x>y$ in $L$. The local dimension of $P$, denoted $\operatorname{ldim}(P)$, is then defined to be the least positive integer 
$d$ for which $P$ has a local realizer $\mathcal{L}$ with $\mu(P, \mathcal{L})=d$.

The concept of local dimension is due to Torsten Ueckerdt who shared his ideas with participants of the workshop on Order and Geometry (held in Gułtowy, Poland, September 14-17, 2016). Clearly, this new notion resonated with participants at the workshop, and it served to stimulate renewed interest in Boolean dimension as well.

Trivially, $\operatorname{ldim}(P) \leqslant \operatorname{dim}(P)$ for all posets $P$. Also, $\operatorname{ldim}(P)=1$ if and only if $P$ is a chain; $\operatorname{ldim}(Q) \leqslant \lim (P)$ if $Q$ is a subposet of $P$; and if $P^{*}$ is the dual of $P$, then $\lim \left(P^{*}\right)=\operatorname{ldim}(P)$. It is an easy exercise to show that if $\operatorname{ldim}(P)=2$, then $\operatorname{dim}(P)=2$. However, for every $d \geqslant 3$, there is a poset $P$ with $\operatorname{ldim}(P)=3$ and $\operatorname{dim}(P)=d$.

The principal result of this paper involves a construction for a family of posets for which local dimension is unbounded. The implications of our construction fall into four distinct categories:

\subsection{Planar Posets}

For two elements $x, y$ of a poset $P$ we say that $y$ covers $x$ if $x<y$ and there is no element $z$ with $x<z<y$. The cover graph of $P$ is the undirected graph $G(P)$ whose set of vertices is the same as the set of elements of $P$, in which $x$ is adjacent to $y$ if and only if $x$ covers $y$ or $y$ covers $x$ in $P$. A drawing on the plane of the cover graph of a poset $P$ is called an order diagram if for any two comparable elements $x<y$ in $P$, the point in the plane corresponding to the element $y$ is higher than the point corresponding to the element $x$. A poset $P$ is planar if its order diagram can be drawn in the plane without edge crossings. If a poset is planar, then its cover graph is planar, although the converse does not hold in general.

For an integer $n \geqslant 2$, the standard example $S_{n}$ is a height 2 poset with minimal elements $A=\left\{a_{1}, a_{2}, \ldots, a_{n}\right\}$ and maximal elements $B=\left\{b_{1}, b_{2}, \ldots, b_{n}\right\}$. Furthermore, $a_{i}<b_{j}$ in $S_{n}$ if and only if $i \neq j$ (see Figure 1). Dushnik and Miller [3] showed that

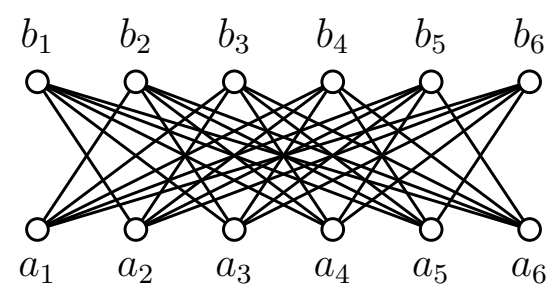

Figure 1: The Standard Example

$\operatorname{dim}\left(S_{n}\right)=n$, for all $n \geqslant 2$ (see [14]). Furthermore, it is an easy exercise to show that $S_{n}$ is planar when $2 \leqslant n \leqslant 4$ and non-planar when $n \geqslant 5$.

In Figure 2, we present a construction due to Kelly [10], showing that for all $n \geqslant 5$, the non-planar poset $S_{n}$ is a subposet of a planar poset $K_{n}$. This specific figure is a diagram for $K_{6}$, but it should be clear how we intend the diagram to be depicted for other values of $n$. 


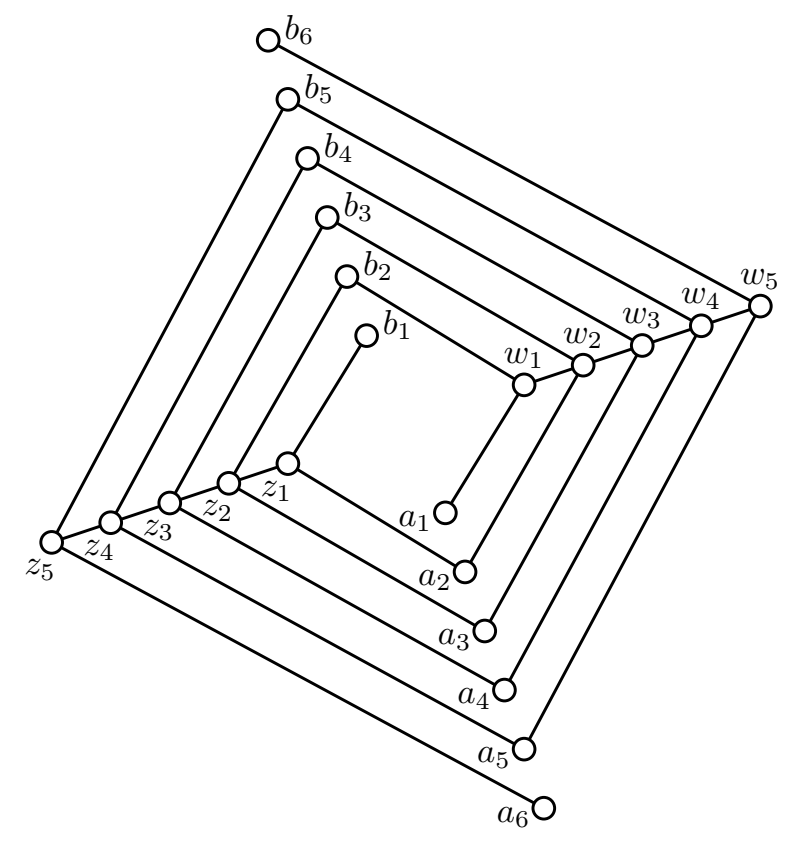

Figure 2: The Kelly Construction

The following argument shows that the bound $\operatorname{bdim}\left(K_{n}\right) \leqslant 4$ holds for all $n \geqslant 3$. First, notice that $\operatorname{dim}\left(K_{3}\right)=3$ implies $\operatorname{bdim}\left(K_{3}\right)=3$. So, assume that $n \geqslant 4$. Then for $K_{n}$ we form linear orders $L_{1}$ :

$$
a_{1}<w_{1}<a_{2}<\cdots<w_{n-1}<a_{n}<b_{n}<z_{n-1}<b_{n-1}<\cdots<z_{1}<b_{1}
$$

and $L_{2}$ :

$$
a_{n}<z_{n-1}<a_{n-1}<\cdots<z_{1}<a_{1}<b_{1}<w_{1}<b_{2}<\ldots<w_{n-1}<b_{n} .
$$

Note that $L_{1}$ and $L_{2}$ are linear extensions of $K_{n}$. Then form linear orders (they are not linear extensions) $L_{3}$ :

$$
a_{1}<b_{1}<a_{2}<b_{2}<\ldots<a_{n}<b_{n}<w_{1}<\ldots<w_{n-1}<z_{1}<\ldots<z_{n-1}
$$

and $L_{4}$ :

$$
z_{n-1}<\ldots<z_{1}<w_{n-1}<\ldots<w_{1}<a_{n}<b_{n}<a_{n-1}<b_{n-1}<\ldots<a_{1}<b_{1} .
$$

Then set $\mathcal{B}=\left\{L_{1}, L_{2}, L_{3}, L_{4}\right\}$, and let $\tau: \mathbf{2}^{4} \rightarrow\{0,1\}$ be defined by setting $\tau(1,1,0,1)=$ $\tau(1,1,1,0)=1$. The map $\tau$ sends all other strings to 0 . It is easy to check that $(\mathcal{B}, \tau)$ is a Boolean realizer for $K_{n}$.

In [12], Nešetřil and Pudlák remarked that the posets in the Kelly construction have Boolean dimension at most 4 , and they asked if the Boolean dimension of planar posets 
is bounded. It is clear from their presentation that they believed the answer should be "yes". However, this challenging question has remained open for nearly 30 years.

For local dimension, Ueckerdt [18] noted that $\operatorname{ldim}\left(S_{n}\right) \leqslant 3$ for all $n \geqslant 2$. In fact, $\lim \left(K_{2}\right)=2$ and $\operatorname{ldim}\left(K_{n}\right)=3$ for all $n \geqslant 3$. Here's why. Suppose $n \geqslant 3$. Let $L_{1}$ and $L_{2}$ be the linear extensions of $K_{n}$ defined just above. Then for each $i=1,2, \ldots, n$, let $M_{i}$ be the ple whose ground set is $\left\{a_{i}, b_{i}\right\}$ with $a_{i}>b_{i}$ in $M_{i}$. Clearly, $\mathcal{L}=\left\{L_{1}, L_{2}\right\} \cup\left\{M_{i}\right.$ : $1 \leqslant i \leqslant n\}$ is a local realizer for $K_{n}$ and $\mu(z, \mathcal{L}) \leqslant 3$ for all $z \in K_{n}$.

In view of these observations, it is also natural to ask whether local dimension is bounded for the class of planar posets. Our construction will show that the answer is "no".

\subsection{Components and Blocks}

We refer the reader to [2] for the concepts of graph theory, including the following terms: connected and disconnected graphs; components; cut vertices; and $k$-connected graphs for an integer $k \geqslant 2$. A block is an inclusion-maximal 2-connected subgraph.

Here are the analogous concepts for posets. A poset $P$ is said to be connected if its cover graph is connected. A subposet $B$ of $P$ is said to be convex if $y \in B$ whenever $x, z \in B$ and $x<y<z$ in $P$. Note that when $B$ is a convex subposet of $P$, the cover graph of $B$ is an induced subgraph of the cover graph of $P$. A convex subposet $B$ of $P$ is called a component of $P$ when the cover graph of $B$ is a component of the cover graph of $P$. A convex subposet $B$ of $P$ is called a block of $P$, when the cover graph of $B$ is a block in the cover graph of $P$.

When $P$ is a disconnected poset with components $C_{1}, C_{2}, \ldots, C_{t}$, for some $t \geqslant 2$, then $\operatorname{dim}(P)=\max \left\{2, \max \left\{\operatorname{dim}\left(C_{i}\right): 1 \leqslant i \leqslant t\right\}\right\}$. Readers may note that the preceding observation is just a special case of the formula for the dimension of a lexicographic sum (see page 23 in [14]). For the local dimension, it is an easy exercise to show that $\operatorname{ldim}(P) \leqslant 2+\max \left\{\operatorname{ldim}\left(C_{i}\right): 1 \leqslant i \leqslant t\right\}$, but we do not know whether this inequality is best possible.

The corresponding result for Boolean dimension is more complicated and is due to Mészáros, Micek and Trotter [11].

Theorem 1. Let $P$ be a disconnected poset with components $C_{1}, C_{2}, \ldots, C_{t}$, for some $t \geqslant 2$. If $d=\max \left\{\operatorname{bdim}\left(C_{i}\right): 1 \leqslant i \leqslant t\right\}$, then $\operatorname{bdim}(P)=O\left(2^{d}\right)$.

The inequality in Theorem 1 cannot be improved dramatically, since it is shown in [11] that for large $d$, there is a disconnected poset $P$ with $\operatorname{bdim}(P)=\Omega\left(2^{d} / d\right)$ and $\operatorname{bdim}(C) \leqslant d$ for every component $C$ of $P$.

The situation with blocks is more complex, even for Dushnik-Miller dimension. In [17], Trotter, Walczak and Wang prove the following result for Dushnik-Miller dimension.

Theorem 2. If $d \geqslant 1$ and $\operatorname{dim}(B) \leqslant d$ for every block of a poset $P$, then $\operatorname{dim}(P) \leqslant d+2$. Furthermore, this inequality is best possible.

Boolean dimension behaves somewhat like Dushnik-Miller dimension with respect to blocks, as the following inequality is also proved in [11]. 
Theorem 3. If $d \geqslant 1$ and $\operatorname{bdim}(B) \leqslant d$ for every block $B$ of a poset $P$, then $\operatorname{bdim}(P)=$ $O\left(2^{d}\right)$.

Again, this inequality cannot be improved dramatically, as it is shown in [11] that for large $d$, there is a poset $P$ with $\operatorname{bdim}(P)=\Omega\left(2^{d} / d\right)$ and $\operatorname{bdim}(B) \leqslant d$ for every block $B$ of $P$.

Our construction will show that local dimension behaves quite differently with respect to blocks. We will prove:

Theorem 4. For every $d \geqslant 1$, there is a poset $P$ such that $\operatorname{ldim}(P)=d$ while $\operatorname{ldim}(B) \leqslant 3$ for every block $B$ of $P$.

\subsection{Structural Graph Theory}

The first major result linking dimension with structural graph theory is due to Joret, Micek, Milans, Trotter, Walczak and Wang [7], who showed that for each pair $(t, h)$ of positive integers, there is a least positive integer $d(t, h)$ so that if $P$ is a poset of height $h$ and the tree-width ${ }^{1}$ of the cover graph of $P$ is $t$, then $\operatorname{dim}(P) \leqslant d(t, h)$. A poset of height 1 is an antichain and has dimension at most 2 , so it is of interest to study $d(t, h)$ only when $h \geqslant 2$. Trotter and Moore [15] showed that $d(1, h)=3$ for all $h \geqslant 2$, and Joret, Micek, Trotter, Wang, and Wiechert [9] showed that $d(2, h) \leqslant 1276$ for all $h \geqslant 2$. Recently, Seweryn [13] has given the following substantive improvement: $d(2, h) \leqslant 12$. It is a relatively simple exercise to show that the posets in the Kelly construction [10] have cover graphs with path-width at most 3 , so $d(t, h)$ goes to infinity with $h$ when $t \geqslant 3$. The best bounds to date in the general case are due to Joret, Micek, Ossona de Mendez and Wiechert [8]:

$$
2^{\Omega\left(h^{\lfloor(t-1) / 2\rfloor}\right)} \leqslant d(t, h) \leqslant 4^{\left(\begin{array}{c}
t+3 h-3 \\
t
\end{array}\right)} .
$$

However, Felsner, Mészáros and Micek [5] proved that the Boolean dimension of a poset is bounded in terms of the tree-width of its cover graph, independent of its height. Formally, here is their result.

Theorem 5. For every $t \geqslant 1$, there is a least positive integer $d(t)$ so that if $P$ is a poset whose cover graph has tree-width $t$, then $\operatorname{bdim}(P) \leqslant d(t)$.

Barrera-Cruz, Prag, Smith, Taylor and Trotter [1] proved that the local dimension of a poset is bounded in terms of the path-width of its cover graph, independent of its height. Formally, here is their result.

Theorem 6. For every $t \geqslant 1$, there is a least positive integer $d^{\prime}(t)$ so that if $P$ is a poset whose cover graph has path-width $t$, then $\operatorname{ldim}(P) \leqslant d^{\prime}(t)$.

However, it is also shown in [1] that the analogue of Theorem 5 for local dimension is false:

\footnotetext{
${ }^{1}$ We refer the reader to [2] for the concepts of tree-width and path-width.
} 
Theorem 7. For every $d \geqslant 1$, there exists a poset $P$ with $\operatorname{ldim}(P)>d$ such that the cover graph of $P$ has tree-width at most 3.

Our construction provides an alternative proof of Theorem 7 .

\subsection{Bounded Boolean Dimension and Unbounded Local Dimension}

Trotter and Walczak [16] proved that if $P$ is a poset and $\operatorname{ldim}(P) \leqslant 3$, then $\operatorname{bdim}(P) \leqslant$ 8442. However, for each $d \geqslant 4$, they also proved that there is a poset $P$ with $\operatorname{ldim}(P)=4$ and $\operatorname{bdim}(P)=d$. They also showed that if $\operatorname{bdim}(P)=3$, then $\operatorname{ldim}(P)=\operatorname{dim}(P)=3$. In [1], it is shown that for each $d \geqslant 1$, there is a poset $P$ with $\operatorname{bdim}(P) \leqslant 4$ and $\lim (P)>d$. Our construction provides another instance of a family of posets where Boolean dimension is bounded and local dimension is not.

\section{Our Construction}

For the remainder of the paper, whenever we discuss a pair $(n, d)$, it will be assumed that $n$ and $d$ are integers with $n \geqslant 2$ and $d \geqslant 1$. Also, we do not distinguish between an element $x$ and a sequence $(x)$ of length 1 .

Fix an integer $n \geqslant 2$. Then for each $d \geqslant 1$, we define a planar poset $\mathrm{K}(n, d)$ via a recursive process. The elements of $\mathrm{K}(n, d)$ will be identified with sequences of length $d$ from the set $\left\{a_{1}, \ldots, a_{n}, b_{1}, \ldots, b_{n}, w_{1}, \ldots, w_{n-1}, z_{1}, \ldots, z_{n-1}\right\}$. As suggested by the notation, the poset $\mathrm{K}(n, 1)=K_{n+1}$, i.e., $\mathrm{K}(n, 1)$ is just the Kelly construction illustrated in Figure 2.

Now suppose that we have defined the planar poset $\mathrm{K}(n, d)$ for some $d \geqslant 1$. Suppose further that we have a planar drawing without crossings of the order diagram of $\mathrm{K}(n, d)$ and that in this drawing, $\left(b_{n}\right)$ is the highest point. To form $\mathrm{K}(n, d+1)$, we take the drawing of $\mathrm{K}(n, 1)$ illustrated in Figure 2 and make the following changes:

For each $i=1,2, \ldots, n$, we take a suitably small scaling of the drawing of $\mathrm{K}(n, d)$ and identify the point $\left(b_{n}\right)$ in $\mathrm{K}(n, d)$ with the point $a_{i}$ in $\mathrm{K}(n, 1)$. We change the label of the points in the copy of $\mathrm{K}(n, d)$ by prepending the symbol $a_{i}$ at the start of the sequence.

With the obvious requirements regarding scaling in mind, it is clear that posets in the family $\mathbb{K}=\{\mathrm{K}(n, d): n \geqslant 2, d \geqslant 1\}$ are planar. We note that for each pair $(n, d)$, a block of the poset $\mathrm{K}(n, d)$ is isomorphic to a subposet of the Kelly construction $K_{n+1}$. Accordingly, if $B$ is a block in $\mathrm{K}(n, d)$, then $\operatorname{ldim}(B) \leqslant 3$.

We also note that posets in the family $\mathbb{K}$ have tree-width at most 3 . Here's why: It is easy to see that the cover graph of the poset $\mathrm{K}(n, 1)$ has path-width at most 3 , so it has tree-width at most 3 . One of the basic properties of tree-width is that the tree-width of a connected graph is just the maximum tree-width of its blocks. Since the blocks of $\mathrm{K}(n, d)$ are isomorphic to subposets of the Kelly construction $K_{n+1}$, they have tree-width at most 3. However, it should be noted that the path-width of cover graphs in the family $\mathbb{K}$ is not bounded.

Let $A=\left\{a_{1}, a_{2}, \ldots, a_{n}\right\}$ and $B=\left\{b_{1}, b_{2}, \ldots, b_{n}\right\}$. The elements of $\mathrm{K}(n, d)$ will be called the core points if they are identified with sequences from $\left(\bigcup_{k=1}^{d} A^{k-1} \times B\right) \cup A^{d}$, 


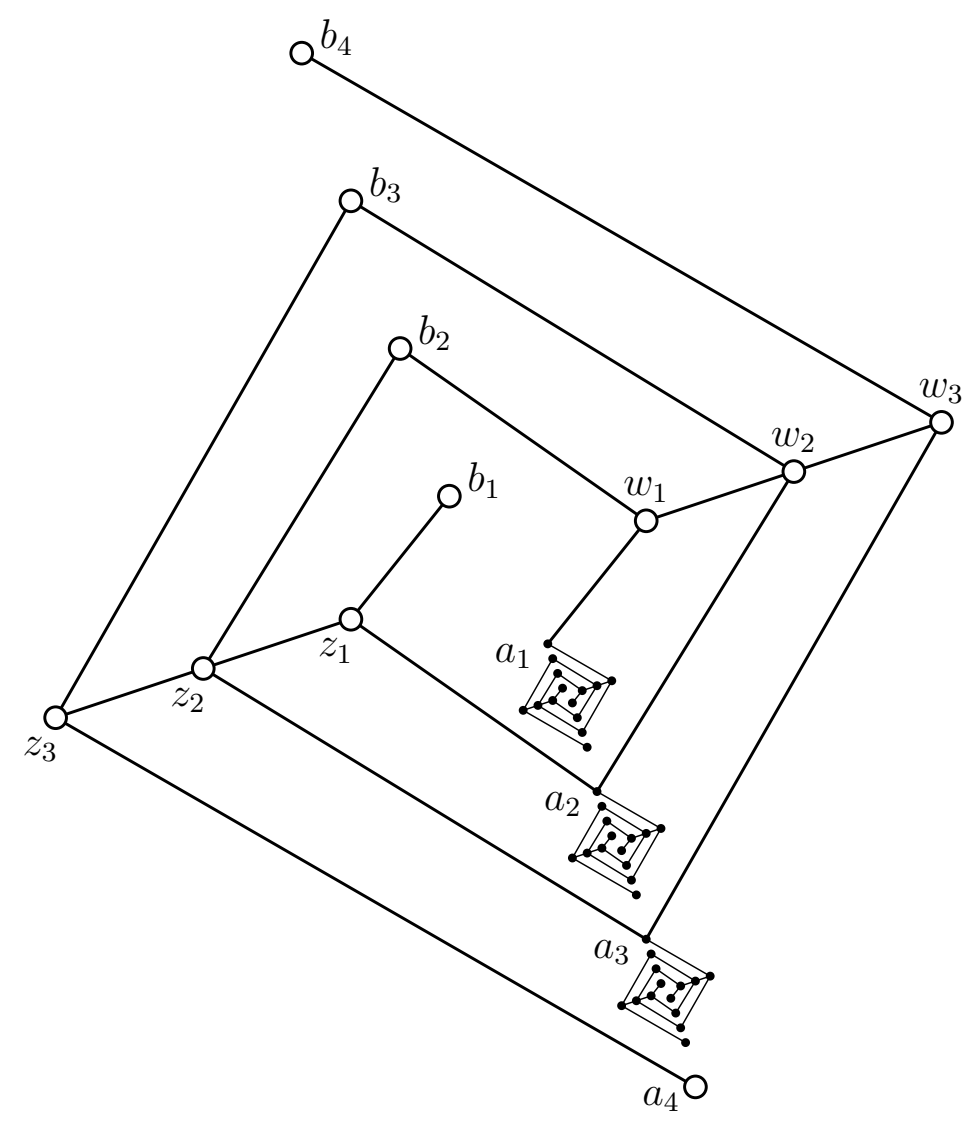

Figure 3: Our construction for $n=3$ and $d=2$

and we will let $\operatorname{Core}(n, d)$ denote the subposet of $\mathrm{K}(n, d)$ determined by the core points. Roughly speaking, the core points are those sequences that consist of $d$ elements of $A$, or less than $d$ elements of $A$ followed by exactly one element of $B$. A subtle detail, that is important later on, is that we are not using $a_{n+1}$ and $b_{n+1}$ in the definition of the $\operatorname{Core}(n, d)$. Note that $\operatorname{Core}(n, d)$ will always be a proper subposet of $\mathrm{K}(n, d)$. Also, note that $\operatorname{Core}(n, 1)$ is just the standard example $S_{n}$.

For the arguments to follow, it is important to understand the structure of the subposet Core $(n, d)$. We state the following elementary proposition for the emphasis.

Proposition 8. Let $u$ and $v$ be two distinct elements of $\operatorname{Core}(n, d)$. Then $u<v$ in Core $(n, d)$ if and only if there is $k \in[d]$ such that $u=\left(a_{i_{1}}, \ldots, a_{i_{k-1}}, a_{i_{k}}, \ldots, a_{i_{d}}\right) \in A^{d}$ and $v=\left(a_{i_{1}}, \ldots, a_{i_{k-1}}, b_{j}\right) \in A^{k-1} \times B$, where $a_{i_{k}}<b_{j}$ in $S_{n}$.

For example, when $n=8$ and $d=6$, we have

$$
u=\left(a_{2}, a_{6}, a_{7}, a_{5}, a_{5}, a_{4}\right)<\left(a_{2}, a_{6}, b_{3}\right)=v
$$

in $\mathrm{K}(8,6)$. Note that $a_{7}<b_{3}$ in $S_{8}$. 
We also note that the subposet $\operatorname{Core}(n, d)$ has height 2. Furthermore, the minimal elements of $\operatorname{Core}(n, d)$ are those sequences of length $d$ with all coordinates in $A$, while the maximal elements of $\operatorname{Core}(n, d)$ are those sequences of length at most $d$ with all coordinates except the last in $A$.

\section{The Local Dimension of Posets in the Class $\mathbb{K}$}

In this section, we will show that the local dimension of posets in the class $\mathbb{K}$ is unbounded. This shows: (1) local dimension is not bounded for the class of planar posets; (2) the local dimension of a poset is not bounded in terms of the maximum local dimension of its blocks; and (3) the local dimension of a poset cannot be bounded in terms of the tree-width of its cover graph, independently of its height. Finally, we will have given another example of a family of posets where Boolean dimension is bounded and local dimension is not.

The proof of our main theorem will require a special case of a result which has become known as the "Product Ramsey Theorem", appearing in the classic text [6] as Theorem 5 on page 113. However, we will use slightly different notation in discussing this result.

Given a finite set $X$ and an integer $k$ with $0 \leqslant k \leqslant|X|$, we denote the set of all $k$ element subsets of $X$ by $\left(\begin{array}{c}X \\ k\end{array}\right)$. When $T_{1}, T_{2}, \ldots, T_{t}$ are $k$-element subsets of $X_{1}, X_{2}, \ldots, X_{t}$, respectively, we refer to the product $g=T_{1} \times T_{2} \times \cdots \times T_{t}$ as a $\mathbf{k}^{t}$-grid in $X_{1} \times X_{2} \times \cdots \times X_{t}$.

Here is a formal statement of the version of the Product Ramsey Theorem we will use in our argument.

Theorem 9. For every 4-tuple $(r, t, k, m)$ of positive integers with $m \geqslant k$, there is a least positive integer $n_{0}=\operatorname{PRam}(r, t, k, m)$ with $n_{0} \geqslant m$ such that if $\left|X_{i}\right| \geqslant n_{0}$ for every $i=1,2, \ldots, t$, then whenever we have a coloring $\phi$ which assigns to each $\mathbf{k}^{t}$-grid $g$ in $X_{1} \times X_{2} \times \cdots \times X_{t}$ a color $\phi(g)$ from a set $R$ of $r$ colors, then there is a color $\alpha \in R$ so that for each $j=1,2, \ldots, t$, there is an m-element subset $H_{j} \subseteq X_{j}$ such that $\phi(g)=\alpha$ for every $\mathbf{k}^{t}$-grid $g$ in $H_{1} \times H_{2} \times \cdots \times H_{t}$.

Actually, we will only use the case where $k=1$, and now the theorem becomes a multidimensional version of the pigeon-hole principle. Readers who would be interested in how this theorem is applied to combinatorial problems on posets when $k \geqslant 2$ are encouraged to consult [1], [4] and [17].

Now we are ready to state and prove our main theorem.

Theorem 10. For every $d \geqslant 1$, there exists a least positive integer $n_{d}$ with $n_{d} \geqslant 2$ so that if $n \geqslant n_{d}$, then $\operatorname{ldim}(\mathrm{K}(n, d)) \geqslant d$.

Proof. The theorem holds trivially for $d \leqslant 2$, with $n_{1}=n_{2}=2$. So for the balance of the argument, we fix a value of $d$ with $d \geqslant 3$. Since Core $(n, d)$ is a subposet of $\mathrm{K}(n, d)$, it suffices to show that $\operatorname{ldim}(\operatorname{Core}(n, d)) \geqslant d$, provided $n$ is sufficiently large. The argument will proceed by contradiction, i.e., we will assume that $\mathcal{L}$ is a local realizer for $\operatorname{Core}(n, d)$ with $\mu(z, \mathcal{L}) \leqslant d-1$ for every $z \in \operatorname{Core}(n, d)$. We will then show that this leads to a contradiction, provided $n$ is sufficiently large. 
We will now describe a recursive procedure which consists of $d$ steps. The procedure will utilize a rapidly growing sequence $\left(p_{1}, p_{2}, \ldots, p_{d}\right)$ of integers with $p_{1}=d$. For the moment, we defer the explanation as to how this sequence is determined, but in time it will be clear that this is done by repeated applications of Theorem 9 .

The key idea of the proof is presented in the following technical claim for which we need to define $A(H)=\left\{a_{h} \in A: h \in H\right\}$ for each $H \subseteq[n]$ and $\operatorname{Min}(X)$ as the set of all minimal elements in $X$.

Claim 11. Let $m \in[d]$ and $p \in \mathbb{N}$. Then there exists $n \in \mathbb{N}$ such that the following statements hold:

1. There are $m-1$ indices $h_{1}, \ldots, h_{m-1} \in[n]$.

2. There exist $d+1-m$ sets $H_{m}, \ldots, H_{d} \subseteq[n]$, with $\left|H_{m}\right|=\cdots=\left|H_{d}\right|=p$.

3. If $\mathcal{L}$ is a local realizer for $\operatorname{Core}(n, d)$, then there exists an $(m-1)$-element family $\mathcal{M} \subseteq \mathcal{L}$ of ple's such that each element in

$$
\left\{a_{h_{1}}\right\} \times \cdots \times\left\{a_{h_{m-1}}\right\} \times A\left(H_{m}\right) \times \cdots \times A\left(H_{d}\right) \subseteq \operatorname{Min}(\operatorname{Core}(n, d))
$$

appears in every ple from $\mathcal{M}$.

It is worth mentioning that for each $i \in[m]$ the value $p_{i}$ equals $p$ from the above claim for $m=d+1-i$ and can be calculated by analyzing the sequence of the calls of the claim in reverse order $m=d, \ldots, d+1-i$.

Proof. We prove the claim by induction on $m \in[d]$. For $m=1$, we must be concerned only about item (2) in this list by setting $n=p$ and $H_{i}=[n]$ for each $i=1, \ldots, d$.

Here's how the inductive step is carried out. We will prove the statement of the claim for $m+1$ and any $p^{\prime} \in \mathbb{N}$. Let us assume that it is true for $m$ and for some fixed $p \in \mathbb{N}$ which depends on $p^{\prime}$. (How big $p$ must be will be described later.) In consequence we obtain an integer $n$, a set $\mathcal{M}$ of ple's, appropriate $h_{1}, \ldots, h_{m-1} \in[n]$, and $H_{m}, \ldots, H_{d} \subseteq[n]$. For each $i=m, \ldots, d$, let $H_{i}=H_{i, 1} \cup H_{i, 2} \cup \cdots \cup H_{i, m}$ be a partition into disjoint subsets with $\left|H_{i, j}\right| \geqslant\lfloor p / m\rfloor$ for all $j=1,2, \ldots, m$. For each $j \in[m]$, we let

$$
\begin{aligned}
U_{j} & =\left\{a_{h_{1}}\right\} \times \cdots \times\left\{a_{h_{m-1}}\right\} \times A\left(H_{m, j}\right) \times \cdots \times A\left(H_{d, j}\right), \\
V_{j} & =\left\{a_{h_{1}}\right\} \times \cdots \times\left\{a_{h_{m-1}}\right\} \times B\left(H_{m, j}\right),
\end{aligned}
$$

where $B(H)=\left\{b_{h} \in B: h \in H\right\}$ for each $H \subseteq[n]$. Note that $U_{j} \subseteq \operatorname{Min}(\operatorname{Core}(n, d))$ and $V_{j} \subseteq \operatorname{Max}(\operatorname{Core}(n, d))$. Also, for each $j=1,2, \ldots, m$, we let $\mathcal{L}_{j}$ denote the subfamily of $\mathcal{L}$ consisting of those ple's which reverse at least one incomparable min-max pair in $U_{j} \times V_{j}$. Note that each $\mathcal{L}_{j}$ is non-empty whenever $p / m \geqslant 1$. We observe that if $1 \leqslant j<j^{\prime} \leqslant m$, then $\mathcal{L}_{j} \cap \mathcal{L}_{j^{\prime}}=\varnothing$. This follows from the fact, that if $(u, v)$ and $\left(u^{\prime}, v^{\prime}\right)$ are incomparable min-max pairs from $U_{j} \times V_{j}$ and $U_{j^{\prime}} \times V_{j^{\prime}}$, respectively, then $u<v^{\prime}$ and $u^{\prime}<v$ in $\operatorname{Core}(n, d)$.

Since $|\mathcal{M}|=m-1$, it follows that there is some integer $j_{0} \in[m]$ so that $\mathcal{M} \cap \mathcal{L}_{j_{0}}=\varnothing$. Let $h_{m}$ be any integer in $H_{m, j_{0}}$ and let

$$
W=\left\{a_{h_{1}}\right\} \times \cdots \times\left\{a_{h_{m-1}}\right\} \times\left\{a_{h_{m}}\right\} \times A\left(H_{m+1, j_{0}}\right) \times \cdots \times A\left(H_{d, j_{0}}\right),
$$




$$
\{v\}=\left\{a_{h_{1}}\right\} \times \cdots \times\left\{a_{h_{m-1}}\right\} \times\left\{b_{h_{m}}\right\} .
$$

It is worth noting that $W \subseteq U_{j_{0}} \subseteq \operatorname{Min}(\operatorname{Core}(n, d))$ and $v \in V_{j_{0}} \subseteq \operatorname{Max}(\operatorname{Core}(n, d))$. Moreover we observe that $v \| w$ in $\operatorname{Core}(n, d)$ for all $w \in W$. It follows that for each $w \in W$, there is some ple $L$ in $\mathcal{L}_{j_{0}} \subseteq \mathcal{L}-\mathcal{M}$ with $w>v$ in $L$. Since there are at most $d-1$ ple's in $\mathcal{L}$ in which $v$ appears, this results in a coloring of the elements of $W$ using at most $d-1$ colors. Since there is a natural one-to-one correspondence between elements of $W$ and $\mathbf{1}^{d-m}$ grids in $H_{m+1, j_{0}} \times \cdots \times H_{d, j_{0}}$, we are then in a position to apply Theorem 9 .

In particular, given a value of $p^{\prime}$, we will assume that $p / m$ is large enough to guarantee that there is a ple $L \in \mathcal{L}_{j_{0}} \subseteq \mathcal{L}-\mathcal{M}$ and a family $K_{m+1}, \ldots, K_{d}$ of sets with $K_{i} \subseteq H_{i, j_{0}}$ and $\left|K_{i}\right|=p^{\prime}$ for each $i=m+1, m+2, \ldots, d$, such that $w>v$ in $L$ for all

$$
w \in\left\{a_{h_{1}}\right\} \times \cdots \times\left\{a_{h_{m}}\right\} \times A\left(K_{m+1}\right) \times \cdots \times A\left(K_{d}\right) \subseteq W .
$$

We then add the ple $L$ to $\mathcal{M}$ and set $H_{i}=K_{i}$ for all $i=m+1, m+2, \ldots, d$.

The final contradiction occurs as we invoke Claim 11 for $m=d$ and $p=d$. Now we have integers $h_{1}, \ldots, h_{d-1}$, a set $H_{d}$ of size $d$, and a subfamily $\mathcal{M}$ of $\mathcal{L}$ with $|\mathcal{M}|=d-1$. It follows that there are exactly $d$ incomparable min-max pairs of the form $(u, v)$ with

$$
\begin{aligned}
& u \in\left\{a_{h_{1}}\right\} \times\left\{a_{h_{2}}\right\} \times \cdots \times\left\{a_{h_{d-1}}\right\} \times A\left(H_{d}\right) \text { and } \\
& v \in\left\{a_{h_{1}}\right\} \times\left\{a_{h_{2}}\right\} \times \cdots \times\left\{a_{h_{d-1}}\right\} \times B\left(H_{d}\right) .
\end{aligned}
$$

Furthermore, these incomparable min-max pairs form a subposet of Core $(n, d)$ which is isomorphic to the standard example $S_{d}$. Because one ple can reverse at most one of the $d$ incomparable pairs, there are (at least) $d$ distinct ple's in $\mathcal{L}$ which reverse these pairs and at least one of these does not belong to $\mathcal{M}$. The $d-1$ ple's from $\mathcal{M}$ with the one outside we just got are witnesses for that, there is some $u$ with $\mu(u, \mathcal{L}) \geqslant d$. The contradiction completes the proof.

\section{References}

[1] Fidel Barrera-Cruz, Thomas Prag, Heather C. Smith, Libby Taylor, and William T. Trotter, Comparing Dushnik-Miller dimension, Boolean dimension and local dimension, Order 37 (2020) 243-269, doi.org/10.1007/s11083-019-09502-6. arXiv:1710.09467.

[2] Reinhard Diestel, Graph theory, fifth ed., Graduate Texts in Mathematics, vol. 173, Springer, Berlin, 2018.

[3] Ben Dushnik and Edwin W. Miller, Partially ordered sets, American Journal of Mathematics 63 (1941), 600-610, doi.org/10.2307/2371374.

[4] Stefan Felsner, Peter C. Fishburn, and William T. Trotter, Finite three-dimensional partial orders which are not sphere orders, Discrete Mathematics 201 (1999), no. 1-3, 101-132, doi.org/10.1016/S0012-365X(98)00314-8. 
[5] Stefan Felsner, Támas Mészáros, and Piotr Micek, Boolean dimension and tree-width, Combinatorica (2020). doi.org/10.1007/s00493-020-4000-9. arXiv:1707.06114.

[6] Ronald L. Graham, Bruce L. Rothschild, and Joel H. Spencer, Ramsey theory, Wiley Series in Discrete Mathematics and Optimization, John Wiley \& Sons, Inc., Hoboken, NJ, 1990, The second edition.

[7] Gwenaël Joret, Piotr Micek, Kevin G. Milans, William T. Trotter, Bartosz Walczak, and Ruidong Wang, Tree-width and dimension, Combinatorica 36 (2016), no. 4, 431450. doi.org/10.1007/s00493-014-3081-8. arXiv:1301.5271.

[8] Gwenaël Joret, Piotr Micek, Patrice Ossona de Mendez, and Veit Wiechert, Nowhere dense graph classes and dimension, Combinatorica 39 (2019), no. 5, 1055-1079. doi.org/10.1007/s00493-019-3892-8. arXiv:1708.05424.

[9] Gwenaël Joret, Piotr Micek, William T. Trotter, Ruidong Wang, and Veit Wiechert, On the dimension of posets with cover graphs of treewidth 2, Order 34 (2017), no. 2, 185-234, doi.org/10.1007/s11083-016-9395-y. arXiv:1406.3397.

[10] David Kelly, On the dimension of partially ordered sets, Discrete Mathematics 35 (1981), 135-156, doi.org/10.1016/0012-365X(81)90203-X.

[11] Támas Mészáros, Piotr Micek, and William T. Trotter, Boolean dimension, components and blocks, Order 37, (2020) 287-298 (2020), doi.org/10.1007/s11083-01909505-3. arXiv:1801.00288

[12] Jaroslav Nešetřil and Pavel Pudlák, A note on Boolean dimension of posets, Irregularities of partitions (Fertőd, 1986), Algorithms Combin. Study Res. Texts, vol. 8, Springer, Berlin, 1989, pp. 137-140.

[13] Michal T. Seweryn, Improved bound for the dimension of posets of treewidth two, Discrete Mathematics 343 (2020), no. 1, 111605. doi.org/10.1016/j.disc.2019.111605. arxiv:1902.01189

[14] William T. Trotter, Combinatorics and partially ordered sets, Johns Hopkins Series in the Mathematical Sciences, Johns Hopkins University Press, Baltimore, MD, 1992, Dimension theory.

[15] William T. Trotter and John I. Moore, The dimension of planar posets, Journal of Combinatorial Theory. Series B 22 (1977), no. 1, 54-67. doi.org/10.1016/00958956(77)90048-x.

[16] William T. Trotter and Bartosz Walczak, Boolean dimension and local dimension, Electronic Notes in Discrete Mathematics 61 (2017), 1047-1053. doi.org/10.1016/j.endm.2017.07.071. arXiv:1705.09167.

[17] William T. Trotter, Bartosz Walczak, and Ruidong Wang, Dimension and cut vertices: an application of Ramsey theory, Connections in discrete mathematics, Cambridge Univ. Press, Cambridge, 2018, pp. 187-199. doi.org/10.1017/9781316650295.012. arXiv:1505.08162.

[18] Torsten Ueckerdt, (2016), personal communication. 